\title{
Consistency Cubes: a fast, efficient method for exact Boolean minimization.
}

by Adrian Dusa

Abstract A lot of effort has been spent over the past few decades in the QCA methodology field, to develop efficient Boolean minimization algorithms to derive an exact, and more importantly complete list of minimal prime implicants that explain the initial, observed positive configurations.

As the complexity grows exponentially with every new condition, the required computer memory goes past the current computer resources and the polynomial time required to solve this problem quickly grows towards infinity.

This paper introduces a new alternative to the existing non-polynomial attempts. It completely solves the memory problem, and preliminary tests show it is exponentially hundreds of time faster than eQMC, the current "best" algorithm for QCA in R, and probes into a territory where it competes and even outperforms engineering algorithms such as Espresso, for exact minimizations.

While speed is not much of an issue now (eQMC is fast enough for simple data), it might prove to be essential when further developing towards all possible temporal orders, or searching for configurations in panel data over time, combined with / or automatic detection of difficult counterfactuals etc.

\section{Context}

QCA (Qualitative Comparative Analysis) is a Boolean minimization method that seeks to find the smallest causal configuration that is associated with (sufficient for) the presence of an outcome of interest. It has been introduced in the social sciences literature by Ragin (1987), and applies an algorithm firmly introduced in the engineering field by McCluskey (1956), building on the previous work of Quine (1952, 1955).

The input for such a procedure is a truth table, which presents all possible combinations of presence (coded with 1) and absence (coded with 0 ) for all causal conditions, plus an additional column to specify in which cases the output is present and respectively absent.

With four causal conditions, a complete matrix with all their possible combinations could be generated using these commands:

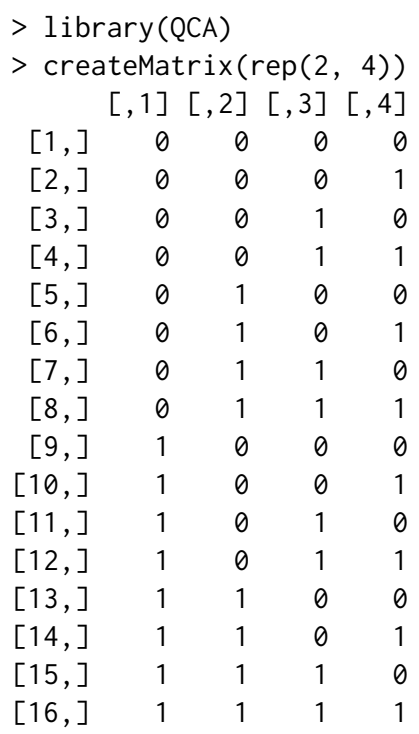

For all rows / combinations of causal conditions that enter the analysis (that is, where an additional column with the output coded as 1), the task is to:

- compare all possible pairs to determine if they can be minimized (only those which differ by exactly one literal)

- compile a list of implicants as the result of minimizing pairs of rows

- iteratively compare all pairs of the surviving implicants and minimize further

- until nothing more can be minimized, thus obtaining the so-called "prime implicants" 
In this example, the last two rows can be minimized (they differ only in the fourth column), with the following result:

\begin{tabular}{llll}
1 & 1 & 1 & 0 \\
1 & 1 & 1 & 1 \\
\hline 1 & 1 & 1 & $x$
\end{tabular}

A computer intensive, iterative process can determine a final solution in a polynomial time for a relatively small number of conditions. But each new condition included in the analysis exponentially increases the complexity of the problem to at least the powers of 3 , and even more for multi-value conditions.

Although the initial truth table is specified in base 2, the calculations are in fact done in base 3 because an additional bit of information is needed to specify which column has been minimized (coded " $x$ " in the example above).

All solutions are found in the so-called "implicant matrix", which for binary crisp conditions has $3^{k}$ rows and $k$ columns, where $k$ is the number of conditions. Solving time increases exponentially with each new condition added in the analysis, and quickly grows towards infinity when $k$ reaches a certain value.

Memory consumption is obviously a big issue, as generating the entire implicant matrix quickly depletes all available memory and make the computer stop because of lack of resources. Such an approach is bound to be inefficient even for moderately sized situations (11 or 12 causal conditions), after which finding a solution in polynomial time becomes impossible.

\section{Previous attempts}

A first attempt to find a non-polynomial complete solution was presented in "A mathematical approach to the Boolean minimization problem" (Dușa, 2010). The basic novelty of that approach was the use of a vector of decimal row numbers instead of the entire implicant matrix. This shortcut partially solved the memory use ( $k$ times lower than a complete matrix), while the solving time was significantly reduced by using a basic mathematical operation $2 x-y$ to minimize two rows using their decimal representations:

\begin{tabular}{lllll}
79 & 2 & 2 & 2 & 1 \\
80 & 2 & 2 & 2 & 2 \\
\hline 78 & 2 & 2 & 2 & 0
\end{tabular}

In this example, the original binary number 0 for the absence is changed to 1 in the base 3 implicant matrix, the original binary number 1 for the presence is changed to the number 2, while the number 0 is reserved to signal a minimized condition. Applying the $2 x-y$ operation, the minimized implicant is found on the line $158-80=78$.

These kinds of improvements helped a lot, at that time obtaining a solution in a significantly lower time and probing into a previously unexplored space of 14 to 15 causal conditions with exact results, the complexity growing three times with each new condition.

However, it was still a problem finding and comparing all possible pairs of minimizable row numbers (not all pairs of numbers can be minimized, because as not all pairs of rows in the implicant matrix differ by exactly one column). A mathematical shortcut was used to find minimizable pairs using the powers of 3 after transforming the row into base 3 , at the cost of more computing time.

Even if using vectors of row numbers instead of matrices, after the ceiling of 14 to 15 causal conditions the memory was still an issue and solving time also progressed towards infinity. In addition, this method was restricted to binary data only, whereas QCA also needed to use multi-valued data.

The next attempt to solve this problem was represented by the so-called "enhanced QuineMcCluskey" procedure (Dussa and Thiem, 2015), which improved on the classical minimization algorithm on both speed and memory, and this algorithm is the current "best" workhorse for QCA family of R packages.

The foundation of the second attempt is represented by an important observation which is also valid for the algorithm to be presented in this paper: the final list of prime implicants are supersets of those cases with a positive output, and at the same time they cannot be supersets of the cases with a negative output. To better understand this concept, the entire truth table can be generated and presented as below: 
$>$ set. $\operatorname{seed}(1234)$

$>$ tt <- data.frame (createMatrix $(\operatorname{rep}(2,4))$, stringsAsFactors = FALSE,

OUT $=\operatorname{sample}(c(1,0, "$ ?"), 16 , replace $=\operatorname{TRUE}, \operatorname{prob}=c(.19, .18, .63)))$

$>\operatorname{colnames}(t \mathrm{t})[1: 4]=\operatorname{LETTERS}[1: 4]$

$>t t$

A B C C D OUT

10000 ?

2000001 ?

$\begin{array}{lllllllll}3 & 0 & 0 & 1 & 0 & \text { ? }\end{array}$

$\begin{array}{lllllllll}4 & 0 & 0 & 1 & 1\end{array}$

5001000

$\begin{array}{llllll}6 & 0 & 1 & 0 & 1 & \end{array}$

$\begin{array}{lllllllllllll}7 & 0 & 1 & 1 & 0 & \text { ? }\end{array}$

8001111 ?

$\begin{array}{llllll}9 & 1 & 0 & 0 & 0 & 1\end{array}$

$\begin{array}{lllllll}10 & 1 & 0 & 0 & 1\end{array}$

$\begin{array}{llllll}11 & 1 & 0 & 1 & 0 & 1\end{array}$

$\begin{array}{lllllll}12 & 1 & 0 & 1 & 1\end{array}$

131100 ?

$\begin{array}{llllll}14 & 1 & 1 & 0 & 1 & 0\end{array}$

151110 ?

1611110

For some rows, the output is positive (coded with " 1 "), for other rows it is absent (coded with " 0 ") while for the rest of the truth table the output is unknown (and coded with "?"). That happens because the number of all possible combinations (especially when the number of causal conditions $k$ increases), is far greater than what we can observe in reality. In other words, as Ragin and Sonnett (2005) put it, the diversity is limited and it can be reduced to this part of the truth table (sorted decreasingly by the output):

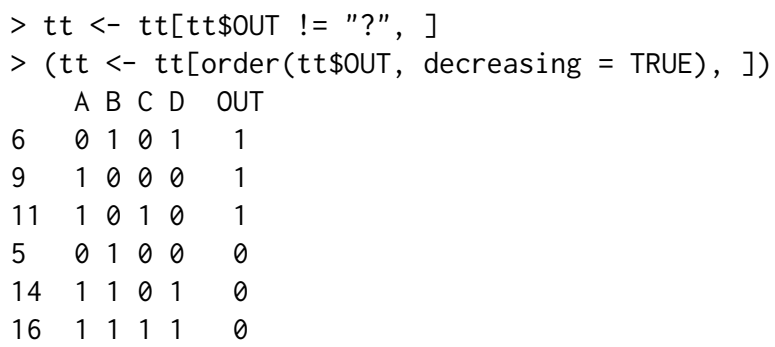

Minimizing the three rows with a positive output is a trivial task and doesn't require a special computer program. The difficulty starts when employing the so-called "counterfactual analysis" to determine the most parsimonious solution, by assuming the output is also positive for the configurations with no empirical information (called "remainders" in QCA, also known as "don't cares").

But instead of calculating all possible pairs of minimizable configurations where the output is not negative (either positive, or unknown, as in the previous attempt), the eQMC algorithm focuses only on the known sub-matrix and applies the following procedure:

- find all possible superset implicants of the rows with a positive output

- find all possible superset implicants of the rows with a negative output

- calculate the differences, preserving those implicants where the output is positive

- remove the redundant implicants: those which already have supersets in the surviving vector of differences

A superset is a simpler, less complex logical expression that covers a specific conjunction. For example, the causal condition $A$ is a superset of the intersection $A^{*} B$. In the implicant matrix, this would be:

$\begin{array}{llllll} & \text { A } & \text { B } & \text { C } & \text { D } & \\ 54 & 2 & 0 & 0 & 0 & A \\ 72 & 2 & 2 & 0 & 0 & A * B\end{array}$

Determining specific supersets of specific row numbers (in this case, that row number 54 is a superset of row number 72) uses the same mathematical tricks as in the previous attempt, employing a vector with the powers of 3 for binary data: $3^{3}, 3^{2}, 3^{1}, 3^{0}$. 
This new procedure brought a great deal of speed compared with the previous one, consuming a lot less memory (for 15 causal conditions, about $15 \mathrm{MB}$ compared to $1.4 \mathrm{~GB}$ ). It also made it possible to explore up to 18 conditions at once, therefore additional three conditions meaning $3^{3}=27$ times more complex situations than before.

Past that threshold, the eQMC algorithm reaches its maximum possibilities. The culprit is less represented by memory consumption, although at an extremely high number of causal conditions the generated vectors of row numbers for the supersets can potentially grow larger than available memory. Possibly problematic, after 19 causal conditions the row numbers cannot even be represented in 32 bit computers, which have an upper representation limit of $2^{31}$, that is still higher than $3^{19}$ but not enough compared to $3^{20}$ for the implicant matrix with 20 causal conditions.

The more serious issue was the calculation time, especially at the final step to remove the redundant implicants from the surviving differences: having that vector sorted in increasing order, the procedure involved an inverse process of finding all subsets for each surviving implicant, starting from the lowest, and iteratively remove those which are found in the rest of the vector.

That process involved a backward transformation from the base 10 row numbers to their base 3 equivalent then applying specific mathematical operations to find all their subsets. For millions or tens of millions of implicants, even this "quick" process can take a lot more time than expected.

\section{Consistency Cubes: the newest approach}

Compared to the first attempt, eQMC excelled because it focused on the observed data only. Indirectly, it probes into the implicants' space during the calculation of supersets and subsets, which requires some memory and costs a lot of time. But the focus on the observed data (ignoring the remainders but obtaining exactly the same solutions), has to be the right approach and still stays at the foundation of the new consistency cubes (CCubes) method, which starts with a simple observation:

Definition 3. A prime implicant is the simplest possible, non-redundant, fully consistent superset of any positive output configuration.

Fully consistent also means that it can never be a superset of any observed negative configuration. The simplest possible superset expression is a single condition (either its presence, or its absence). Figure 1 presents the graphical representation of the generated truth table $t t$ above, and shows that no single condition qualify as a prime implicant in its presence (lit cubes above and below the middle separator). The only condition that is fully consistent in its absence is B, but since it does not cover all observed positive configurations, the search must be continued at the next level of complexity.

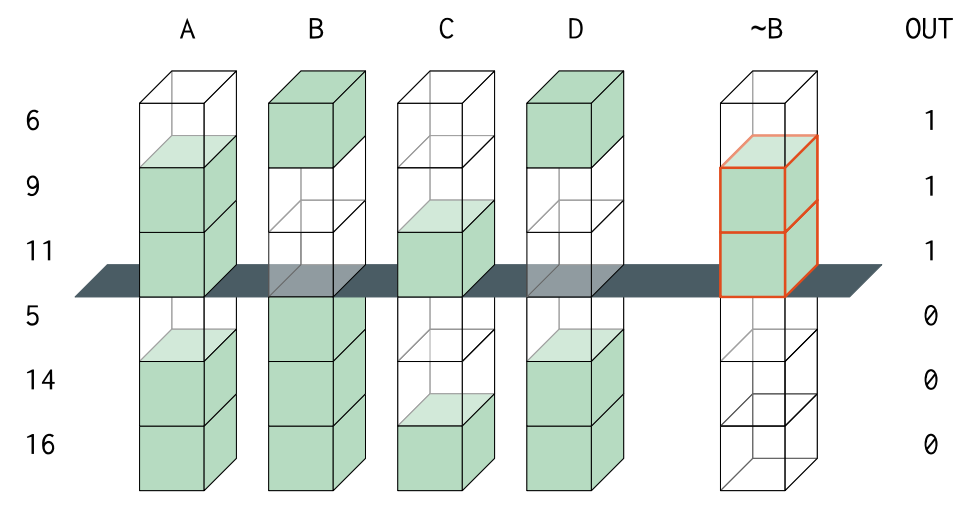

Figure 1: Individual presence inconsistencies, absence of B consistent

The next simplest superset expressions can be found in all combinations of 2 conditions out of 4 , and for each such combination a normal computer program would search within all pairs of their respective levels. If conjunctions of two conditions will not prove to be sufficient, the next simplest are superset expressions formed with 3 conditions out of 4 , and so on.

For any general case involving multi-value causal conditions, the complete search space $S$ (containing the total number of possibilities to check using a polynomial approach) is equal to the sum of all combinations of $c$ selected conditions out of $k$, times the product of their respective number of levels $l_{s}$ for each such selected combination:

$$
S_{M V}=\sum_{c=1}^{k}\left(\begin{array}{l}
k \\
c
\end{array}\right) \prod_{s=1}^{c} l_{s}
$$


The CS (crisp sets) space is just a special case with all binary causal conditions having two levels each, 0 and 1, where this equation gives the same result as $3^{k}-1$ that describes the implicant matrix having exactly this number of all possible expressions which Ragin (2000, pp.132-136) calls "groupings". These are all rows from the entire $3^{k}$ implicant matrix, minus the first one which indicates a situation when all causal conditions are minimized:

$$
S_{C S}=\sum_{c=1}^{k}\left(\begin{array}{l}
k \\
c
\end{array}\right) 2^{c}=3^{k}-1
$$

Ragin uses a similar technique and lists all possible combinations of causal conditions and their levels in Table 5.4 (p.134). But he applied his calculation backwards, starting from the most complex expressions of four conditions out of four, towards all combinations of levels from the most simple situations with one separate condition at a time.

As it will be shown in the next section, starting from the simplest expression towards the most complex, has a direct consequence over the performance of this algorithm. Fast forward, the properties of Equation 1, combined with the requirements from Definition 3 that describe a minimal prime implicant, are key ingredients behind the speed of the new CCubes algorithm.

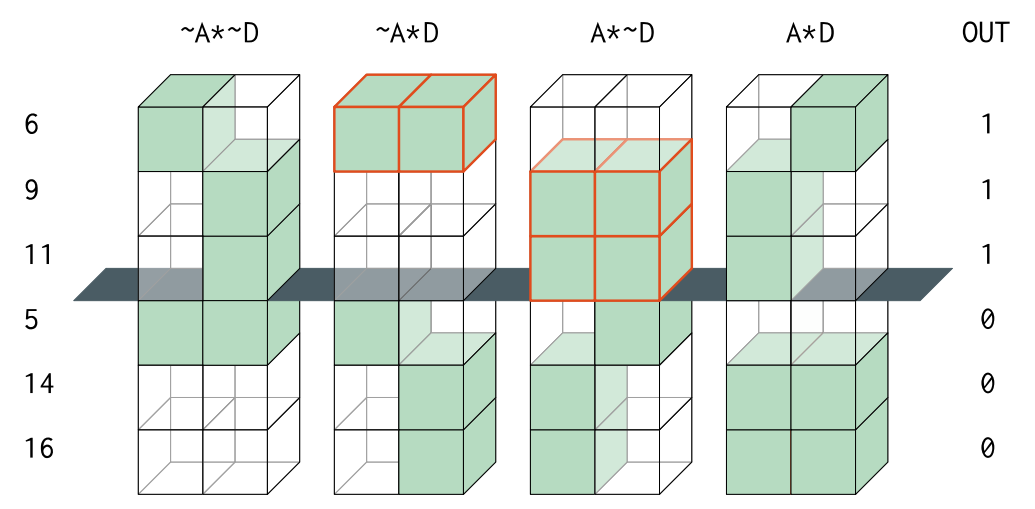

Figure 2: Conjunctive prime implicants

Increasing the complexity to level $c=2$, Figure 2 above shows all possible combinations of levels for the pair of conditions $A$ and $D$, out of which two qualify as prime implicants: $\sim A * D$ and $A * \sim D$ are both associated with a positive output above the middle grey separator, and at the same time not associated with a negative output below the separator.

\section{Search space}

An exhaustive exploration of the entire search space, through all possible combinations of columns, and all their subsequent combinations of levels, is a polynomial approach which is bound to consume time. Figure 2 displays all combinations of levels from columns A and D, but there are 6 such pairs of columns, each having 4 pairs of levels. For each pair of columns, a basic program would have to spend time comparing 2 columns, times 4 pairs of levels, times 6 rows which gives 48 computer cycles.

The search space described in Equation 1 can be partitioned into successive layers of complexity, from a single condition for $c=1$ to the most complex conjunctions for $c=k$. Within each layer, it is not necessary to search within all possible combination of levels, because the relevant ones can already be found in the observed data. This is a fortunate situation that alleviates the memory problem because, unlike the previous two attempts, no other memory needs to be used in the process.

The particular arrangement of the cube components in Figure 2 offers a first hint: out of the four pairs, only two are meaningful, and they need not be displayed into separate combinations of levels, as they can already be detected in the original structure of the observed data.

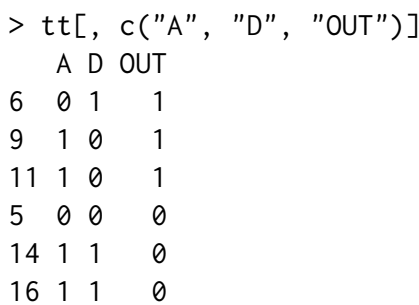


The conjunction $\sim A * D$ (which is the binary combination ' 01 ', on the first line number 6 ), and the conjunction $A * \sim D$ (binary combination ' $10^{\prime}$ ' on the second and third lines, numbers 9 and 11) are both prime implicants because they are found in the observed positive cases and not in the negative ones.

In this example, the search space can be shortened to only two columns times 6 rows, already four times quicker than a polynomial approach. But as it turns out, the search space can be shortened even further using the mathematical tricks from the previous approaches, transforming from any (multiple) base (in this example with all conditions in base 2) to their decimal representation:

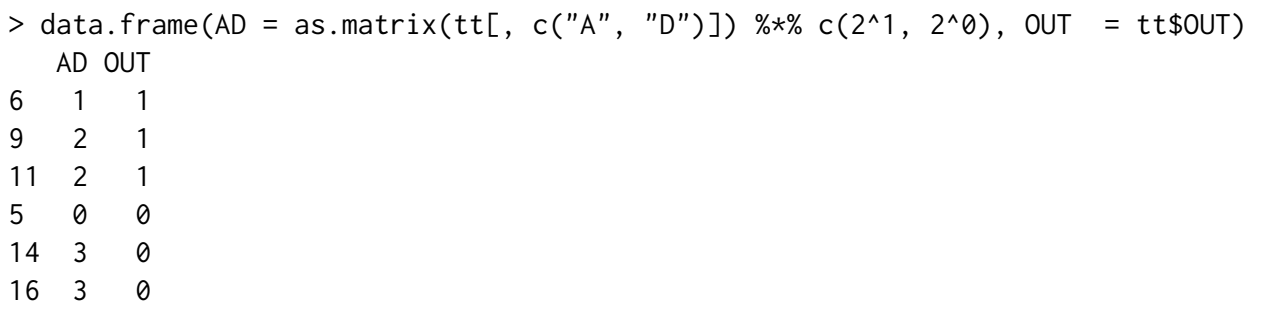

Instead of the exhaustive 48 computer cycles, the same conclusion can be derived by examining only these 6 rows, where decimal numbers 1 and 2 (corresponding to the binary combinations 01 and 10) are not found among the observed negative cases. In this simple example, the gain might be minimal, but for more complex datasets with conjunctions of 5 or 6 causal conditions, these sort of transformations dramatically reduce the search space, with a significant boost of the overall speed.

This technique of partitioning the search space is fundamentally different from the previous two attempts, which approached the implicant matrix in the increasing order of its decimal row numbers. The implicant matrix is by definition unpartitioned, and the example below display all levels for each individual condition, equivalent to the simplest level of complexity in Figure 1:

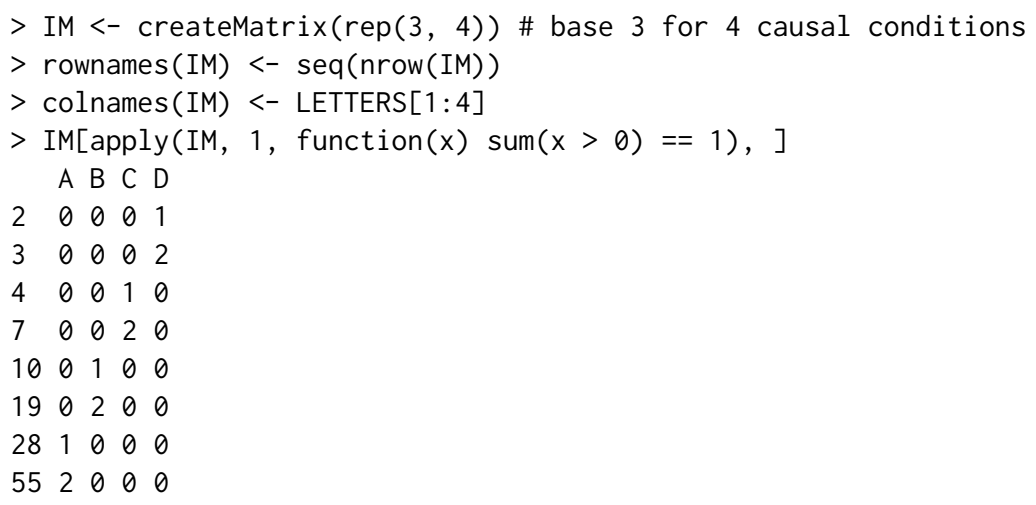

The presence of a single condition A is found all the way on the decimal row number 55, therefore the entire implicant matrix has to be scanned to find even the most simple conjunctions, and that takes a lot of time. By contrast, and in line with Definition 3, the same search space can be partitioned in successive layers of complexity before starting the search process, as shown in Equation 1.

This is one of the key novelties of the CCubes algorithm, that boosts its speed to unprecedented levels while maintaining the same overall requirement to arrive at exact and complete solutions. It does that by exploring the same space (the implicant matrix), but using a different method. As an added bonus, the new algorithm uses the best features from all attempts, by partitioning the search space and at the same time using the decimal representation from eQMC, when comparing which numbers are found in the positive part and (not) in the negative part of the truth table.

\section{Search depth}

Another factor that can increase the speed of the CCubes algorithm is the search depth, which seeks to terminate the partitioned search space as soon as possible. To satisfy Definition 3 , the algorithm has to search through all possible combinations of $c$ selected conditions out of the total $k$, starting from a single condition with $c=1$ up to the most complex conjunctions with $c=k$.

But parsimonious prime implicants are never found among the most complex expressions, instead they are always found among the simplest possible ones. A "simple" expression, for say 4 or 5 initial causal conditions is a single condition or a conjunction of two conditions. For a more complex dataset with 20 causal conditions, the word "simple" has a different meaning, with possible conjunctions of up to 4 or 5 conditions each. 
More complex expressions are either not contributing to the minimization, or they are subsets of other, less complex expressions which have already been found in the previous iterations of c selected conditions. As the redundant prime implicants are to be removed anyways (a key part of the current eQMC procedure), they will never qualify as "minimal" prime implicant expressions. This is why Definition 3 explicitly mentions "non-redundant" expressions that do qualify as prime implicants.

To maximise speed, the search depth has to be stopped as soon as there is proof that no more prime implicants will be found at more complex expressions. A brute force traditional algorithm searches the entire search space from equation 1 . In the binary crisp sets analysis, it involves an exhaustive search of all $3^{k}$ possible combinations in the implicants matrix. But that is a huge space, especially at a large number of causal conditions, and most of the verifications are void. It is one of the reasons why the traditional algorithms are inherently slow.

A brute force, exhaustive search will always find all non-redundant prime implicants, at the cost of a larger search time. The task is to find those prime implicants without spending additional time for unnecessary, void verifications. As it turns out, the design of the equation 1 is also the answer for this task.

Using the truth table tt presented in this paper, any minimization algorithm will find exactly four prime implicants: $\sim B, \sim A \star D, A * \sim D$ and $C * \sim D$, extracted from an implicants matrix with $3^{4}-1=80$ rows.

The simplest prime implicant is $\sim B$, which has the biggest number of subsets in the implicants matrix. More complex prime implicants have fewer subsets, and there is a method to determine the exact number of subsets for any given prime implicant.

The prime implicant $\sim B$ can be written as 0100 (where 1 means absence of $B$, and 0 means all other conditions are minimized), and using a similar product formula as that from equation 1 it can be determined it has $27-1=26$ subsets. There are three minimized conditions in this prime implicant (A, $C$ and D), each having $2+1$ levels in the implicants matrix, and the number of subsets can be obtained with the product of the number of levels for the minimized conditions:

$$
\prod_{c=1}^{3}\left(l_{c}+1\right)=3^{3}=27
$$

27 rows from the implicant matrix contain $\sim B$, out of which 26 are subsets for which further verifications would consume unnecessary time. The second prime implicant $\sim A \star D$ has 8 subsets, out of which 3 are already covered by $\sim B$, making 31 unique subsets. The third has 8 subsets as well, out of which 3 are already covered by the first two, making 36 unique subsets, and the fourth has 8 subsets out of which 5 are already covered by the first three, making a grand total of 39 unique subsets for all found prime implicants.

Out of the 80 rows of the implicants matrix to verify, it is certain that 39 will turn void results, which means the search could have been terminated after about half of the search space. This procedure is valid for any number of input causal conditions and for any number of non-redundant prime implicants, leading to the general formula:

$$
S_{M V}=\sum_{c=1}^{k}\left(\begin{array}{l}
k \\
c
\end{array}\right) \prod_{s=1}^{c} l_{s}=P+U+N
$$

Here $P$ is the number of prime implicants, $U$ is the total number of unique subsets (of all found $P$ prime implicants), and $N$ is the number of non (prime) implicants. The new algorithm verifies all $P$ rows, most of the $N$ rows, and a small percentage of the $U$ unique subsets. It stops when there is proof that all remaining rows are either $U$ or $N$ : when the prime implicants chart can be solved at a certain complexity level $c$, and no more prime implicants are identified at complexity level $c+1$.

This is a crude, but very effective rule to make sure the search is exhaustive, although only a fraction of the entire implicants matrix is actually verified. $U$ represents the largest part of the implicants matrix, and is not necessary to be verified. Avoiding the search through this void part of the implicants matrix dramatically increases the speed of the CCubes algorithm.

To find all possible $P$ prime implicants in a shorter time is already a major achievement, but the next section presents a method to stop the search even sooner, for the default option of an absolute minimal solution corresponding to the setting min.pin = TRUE in function minimize().

The prime implicants chart gives a better understanding of how many prime implicants are needed to cover all observed positive cases. By iteratively solving this chart after each partition of complexity, the search space can be terminated as soon as the number of prime implicants needed to solve the chart reaches a minimum. 


\section{Solving the prime implicants chart}

Once the prime implicants are found, the next and final step of the Quine-McCluskey procedure is to find the minimal disjunctions of expressions (sums of products) that cover all the initial positive configurations in the observed data. The hypothetical example above generates four prime implicants:

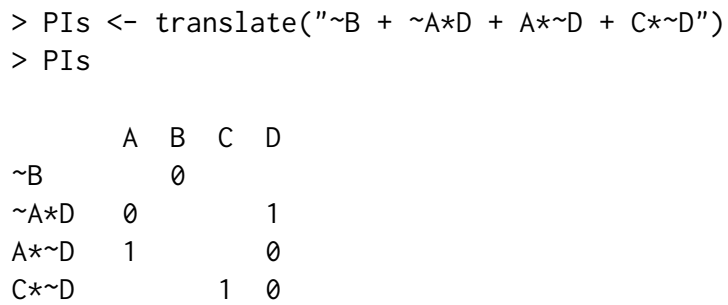

The PI chart is obtained by distributing these four prime implicants on each of the three observed, positive configurations. In this chart representation, the symbol $\mathrm{x}$ indicates the prime implicant is a superset of a specific initial configuration (it covers a specific column) and the symbol - indicates the prime implicant is not a superset of (does not cover) that specific configuration:

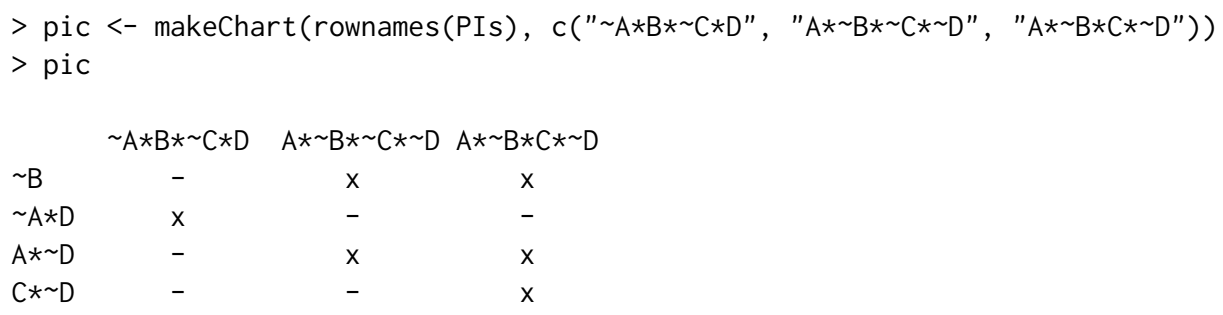

None of the generated prime implicants is able to explain all three initial configurations, because none is covering all three columns. The final solution will be a disjunction of at least 2 prime implicant expressions, where $\sim A * D$ is an essential part of the solution because it is the only one which covers the first column.

The expression $C \star \sim D$, although minimal and non-redundant, is found to be irrelevant because it covers a column which is already covered by two other prime implicants (the so called principle of "row dominance"): both $\sim B$ and $A \star \sim D$ dominate $C * \sim D$ because they cover more columns.

The final solution is a disjunction of exactly 2 prime implicants, and there are two alternative such solutions:

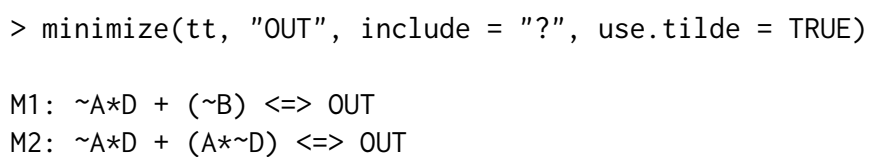

The package QCA (Dușa, 2019; Thiem and Dușa, 2012) in R (R Core Team, 2017) used to have a dependency on package lpSolve, to determine the minimum number of prime implicants through linear programming. There is, however, another direct method of determining this minimum number, involving a two phase procedure:

1. iteratively simplify the chart, by:

- eliminating dominated rows

- eliminating dominating columns

2. iteratively find all sets of (the rest of the) rows that maximally cover the (rest of the) columns

On each iteration in the first phase, the chart is progressively simplified, and on each iteration in the second phase, a new prime implicant is introduced to each previous maximal set, and the procedure stops with the first set to win and cover all columns. There is a dedicated function to find this minimum number $\mathrm{m}$, equal to the output of the previous linear programming:

$>$ findmin(pic)

[1] 2

For this particular purpose, the new procedure is faster than linear programming but the gain is negligible. The real purpose is to solve the prime implicants chart from within the CCubes algorithm after each partition of complexity c out of $k$ from Equation 1, to determine: 
- if the generated prime implicants can explain all initial positive configurations (all columns in the prime implicants chart are covered by at least one row)

- more importantly, if the number of prime implicants necessary to explain all positive configurations reaches a minimum (when at complexity $c+1$ the number $m$ remains the same).

These factors terminate the search space at the depth where the new prime implicants do not contribute to minimally solving the PI chart, thus demonstrating that not all prime implicants are relevant. Only those which are found at the lowest levels of complexity from the search space matter.

This is rather easy to explain: the more complex a prime implicants is, the more specific it becomes and covers fewer columns from the PI chart (initial combinations). A more and more specific conjunction is a smaller and smaller intersection that covers less and less from the Universe, as shown in the following Venn diagram using the package venn (Dusa, 2018):
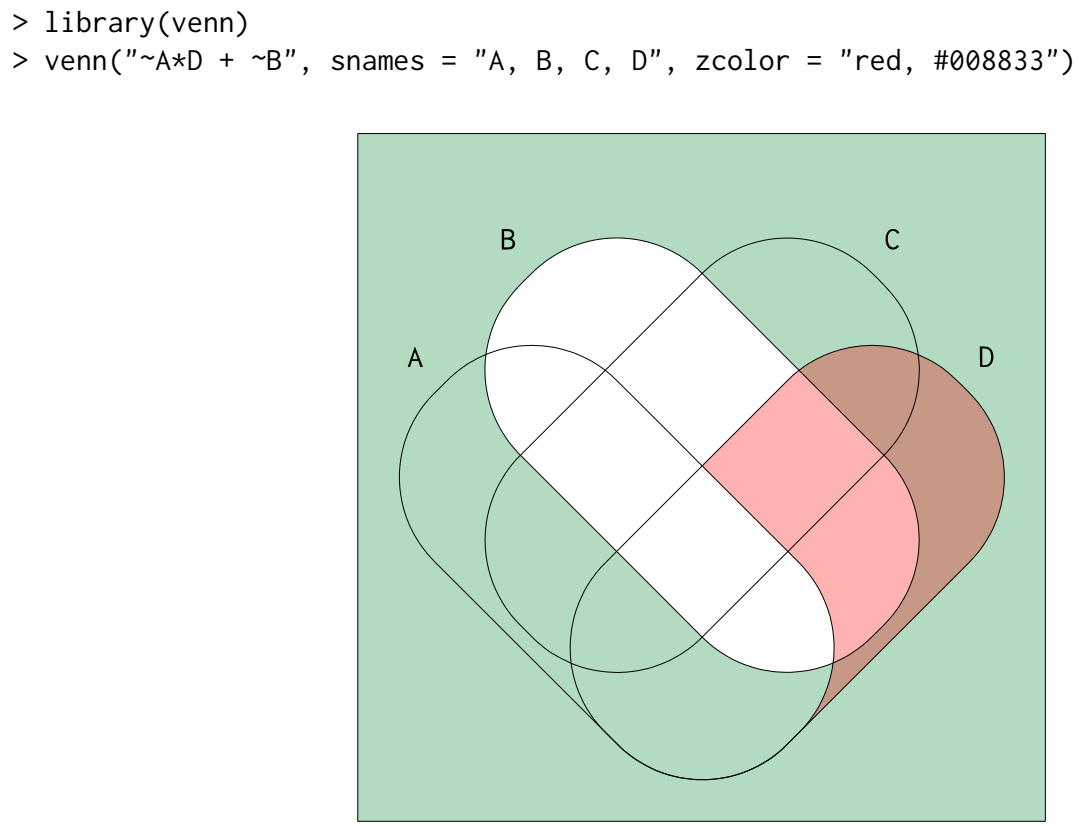

Figure 3: Venn diagram with coverage of $\sim A * D$ compared to $\sim B$

Figure 3 above shows the green area corresponding to $\sim \mathrm{B}$ of complexity level 1 , is much larger than the red area corresponding to $\sim A * D$ of complexity level 2 . A more specific intersection, for example $\sim A \star C \star D$ at complexity level 3 , covers an even smaller area (it is actually a subset of $\sim A \star D$ ).

Less complex prime implicants have a much higher chance of covering more columns from the prime implicants chart, which explains why the most complex PIs are almost never part of the minimal solution. For 20 causal conditions, tests have revealed that prime implicants are found up to the complexity level 7, while the minimal solutions don't use prime implicants from a complexity level more than 4.

This is an important observation: although non redundant PIs can be found at higher complexity levels, the search can be stopped at lower complexities, as soon as there is proof the prime implicants chart is solved using a minimal number of prime implicants. Terminating the search space at lower complexity levels dramatically shortens the time necessary to find all possible minimal solutions.

\section{Performance benchmark}

All three algorithms (QMC, eQMC and CCubes) are now available in function minimize() via the new argument method. The motivation is partly due to the existence of multiple competing algorithms and users should choose which one to use, but more importantly to allow for testing and benchmarking, controlling for input and return exactly the same output. The main difference maker is the speed.

In the world of Boolean minimization, the acknowledged champion software is Espresso (Brayton et al., 1984), built more than three decades ago at the University of California, Berkeley. It is a heuristic algorithm that finds a minimal solution with record speed, but unsuitable for social science research. While in circuit design the goal is to find one minimal solution, QCA needs to find all possible causal paths that are sufficient for an outcome, thus involving all possible prime implicants that form a PI chart. 
The output of interest for all three algorithms is the list of prime implicants, either as a matrix for QMC and CCubes, or as a numerical vector of corresponding row numbers for eQMC. The output itself is therefore not identical, but the final solutions are. What matters here is the timings, on a MacBook Pro with an Intel Core i7 processor at $2.7 \mathrm{GHz}$ with 16GB of RAM, at $1600 \mathrm{MHz}$ DDR3.

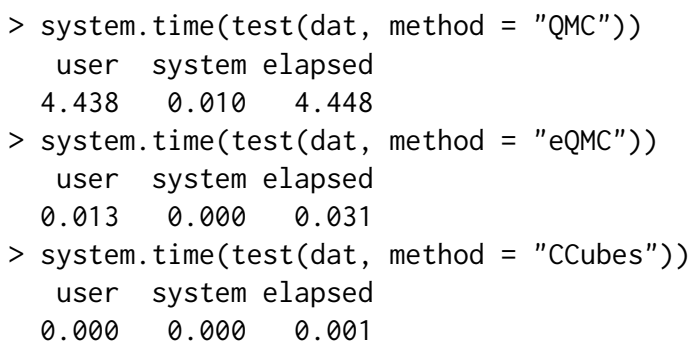

For as little as 10 causal conditions, the classical Quine-McCluskey algorithm is already the slowest, while eQMC performs very decent. The real test begins 16 conditions and above:

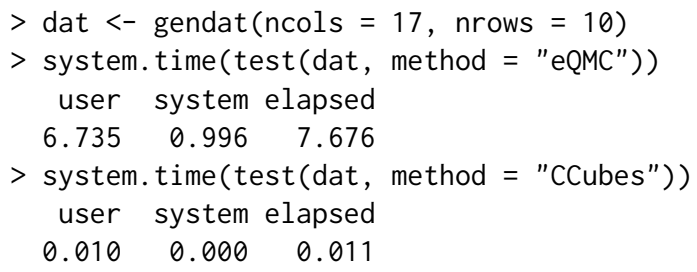

Past 18 causal conditions, the eQMC algorithm reaches its upper limit, while the CCubes algorithm performs effortlessly:

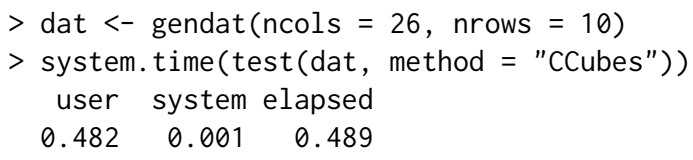

Obviously, some truth tables are more complex, the speed depending not only on the number of causal conditions but also on the number of input configurations and their complexity. In this example, the truth table is a very simple one with 5 positive and 5 negative configurations, but more unique configurations added to the truth table have an obvious impact, even with fewer causal conditions:

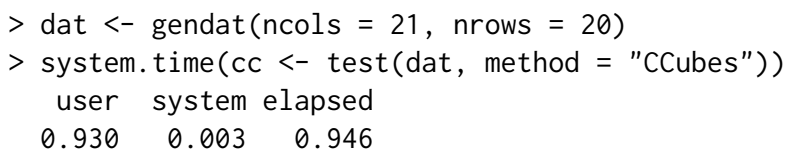

Comparing with Espresso is the more serious challenge. Despite its heuristic nature, it also has a particular implementation that can find all relevant prime implicants, known in the literature under the name of "Espresso-exact" and adapted in the package LogicOpt for comparison with package QCA. Due to historical reasons, Espresso-exact generates not one set of prime implicants, but three: EPs - essential prime implicants, PRPs - partially redundant prime implicants and TRPs - totally redundant prime implicants.

The relevant function in package LogicOpt is called logicopt(), with a control argument named mode that can take three values: "primes" (which leverages the PI generation C-code that is a part of "Espresso-exact"), "multi-min" (which finds the minimum set of non-redundant solutions that cover all the EPs and the minimal set of PRPs) and "multi-full" (that finds additional coverings of prime implicants beyond what is found in multi-min, and an exhaustive covering of all PRPs). However, since the number of coverings (solutions) is potentially very large, the package LogicOpt employs an arbitrary upper limit of 50 coverings, therefore the output of these two arguments is not directly comparable with the exhaustive number of solutions found by package QCA.

The only way to compare with Espresso-exact is to employ the argument mode = "primes" and measure the time taken to identify the prime implicants.

Out of the three sets of prime implicants from Espresso-exact, only the EPs and PRPs play an active role in finding the minimal solutions when solving the PI chart, however it's implementation spends additional time to find the set of TRPs. On the other hand, CCubes does not bother with the TRPs and finds only the relevant set of PRPs (the essential PIs are identified when solving the PI chart). 


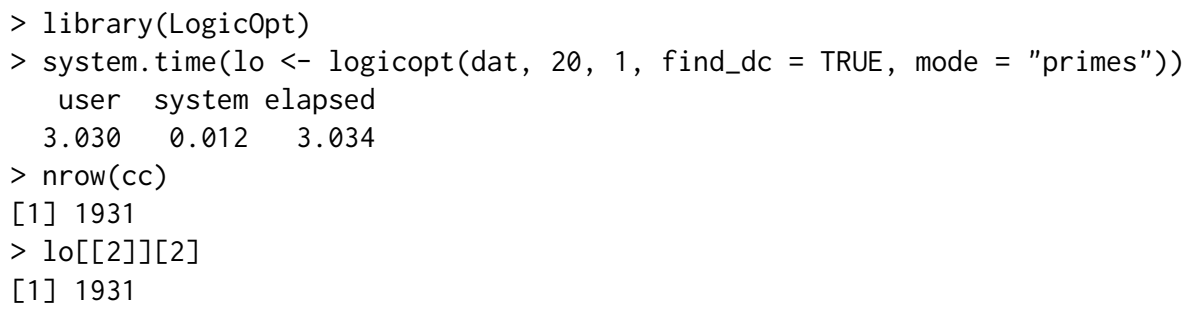

There are exactly 1931 prime implicants identified by both Espresso and CCubes, which is a cross-validation proof between these two algorithms. In terms of speed, CCubes seems to be faster and it can be faster still, by choosing to stop the search when the PI chart can be solved with a minimal number of PIs, using the argument min. pin:

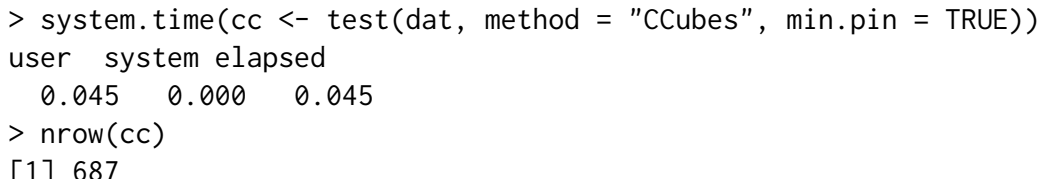

Out of the 1931 prime implicants, only 687 really contribute to minimally solving the PI chart, with the search being terminated a lot sooner with the same final solutions. A more intensive test of speed was carried by generating 1000 random datasets changing the seed in function gendat ():

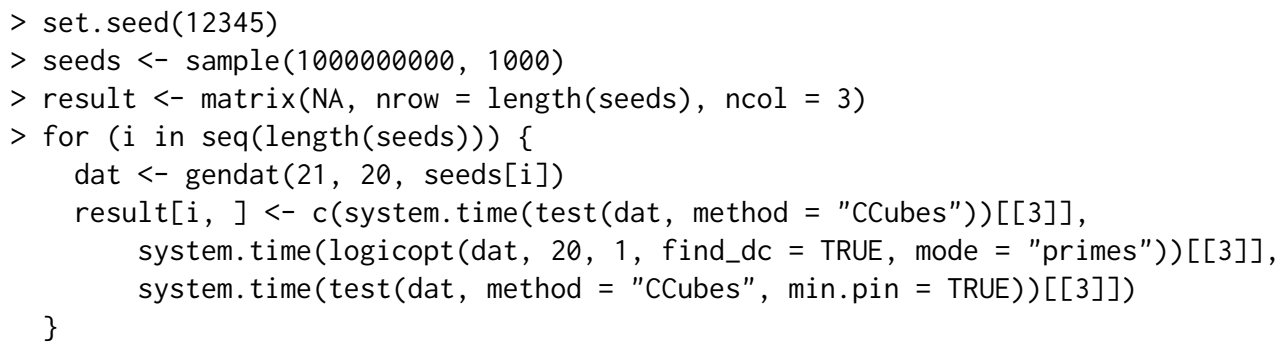

On average, CCubes spends 0.941 seconds per dataset, while Espresso-exact spends 2.017 seconds. The timings are more homogeneous for CCubes, with a standard deviation of 0.550 seconds, compared to 1.727 seconds for Espresso-exact. Using the argument min.pin, CCubes spends an impressive average of 0.571 seconds (over 3.5 times faster than Espresso-exact). In some cases it can be thousands of times faster, while slower in only $5.7 \%$ of cases, depending on how many complex PIs contribute to solving the PI chart (more time spent when approaching the total number of PIs).

While the cross-validation between Espresso-exact and CCubes lies in the number of found PIs, the purpose of the QCA minimization is to make sure all possible (minimal) solutions are found. From this perspective, CCubes is the only algorithm in $\mathrm{R}$ that can do that out of the box, but it is not the only one to operate with a high number of causal conditions.

Another software that is capable to output complete and exhaustive solutions is called Tosmana (Cronqvist, 2017), that implements another very different algorithm called GBA - Graph Based Agent. A direct comparison is not possible in this paper because Tosmana does not operate in $\mathrm{R}$, but according to all tests the solutions are identical, with a superior speed for CCubes.

A cross-validation with QMC (up to 10-11 conditions), eQMC (up to 16-18 conditions), Tosmana (around 20 conditions) and Espresso-exact (up to 30 conditions but indirectly via the number of prime implicants) should be sufficient for any QCA researcher seeking a confirmation that CCubes and the other algorithms used in package QCA are exact and exhaustive.

\section{Summary}

A cube is understood as a multi-dimensional matrix. Just as sets can be either subsets or supersets of other sets, cubes can sometimes be formed with more complex, and sometimes with less complex cubes. The cube should be defined and interpreted relative to a certain context: it can be either a single condition, or a conjunction of causal conditions, or the entire dataset where that conjunction was extracted from. Figure 2 offers a graphical exemplification of a "cube": the simplest structure can be one single red colored component, but if thinking about an entire conjunction as a "single" component, then $\sim A \star D$ can be considered a cube as well, and the entire structure where this particular conjunction resides can also be considered a cube. 
Such a conceptualization of a cube is important for the social sciences and it can be even better understood by noting that, at its heart, the CCubes algorithm is only an extension of Mill's joint method of agreement and difference:

\begin{abstract}
If two or more instances in which the phenomenon occurs have only one circumstance in common, while two or more instances in which it does not occur have nothing in common save the absence of that circumstance; the circumstance in which alone the two sets of instances differ, is the effect, or cause, or a necessary part of the cause, of the phenomenon. John Stuart Mill (1843) A System of Logic, vol. 1, pp. 463.
\end{abstract}

Usually, researchers think about a "circumstance" as a single causal condition, but a conjunction of causal conditions can also be considered a circumstance, or in this case a "cube". Instead of highly expensive, base to base mathematical transformations, this new algorithm merely applies the fundamentals of the comparative method.

It seems that, after all, simple is better. And a whole lot quicker.

\title{
Acknowledgements
}

This new algorithm appeared while preparing a presentation for the Logic Seminar, organised at the Faculty of Mathematics and Informatics, University of Bucharest. The author is grateful to all participants of that Seminar, and especially to Professor Ioana Leustean and students Irina Gutanu and Matei Bejan, for their valuable ideas and fine tuning my $\mathrm{C}$ programming. 2017.

The paper was also presented at the 5th International QCA Expert Workshop in Zürich, December

Special thanks go to Lasse Cronqvist and to William Stiehl for their generous time while attempting to cross-validate the results, a number of important ideas from this paper being generated during this process.

\section{Bibliography}

R. K. Brayton, A. L. Sangiovanni-Vincentelli, C. T. McMullen, and G. D. Hachtel. Logic Minimization Algorithms for VLSI Synthesis. Kluwer Academic Publishers, Norwell, MA, USA, 1984. ISBN 0898381649. [p365]

L. Cronqvist. TOSMANA - TOol for SMAll-N Analysis (version 1.54). University of Trier, 2017. URL http://www. tosmana. net. [p368]

A. Dusa. A mathematical approach to the boolean minimization problem. Quality $\mathcal{E}$ Quantity, 44(1): 99-113, 2010. URL https://doi.org/10.1007/s11135-008-9183-x. [p358]

A. Dușa. Venn: Draw Venn Diagrams, 2018. URL https://CRAN.R-project.org/package=venn. R package version 1.7. [p365]

A. Dușa. QCA with R. A Comprehensive Resource. Springer-Verlag, New York, 2019. URL https: //doi.org/10.1007/978-3-319-75668-4. [p364]

A. Dușa and A. Thiem. Enhancing the minimization of Boolean and multivalue output functions with eQMC. Journal of Mathematical Sociology, 39:92-108, 2015. URL https://doi .org/10.1080/ 0022250x.2014.897949. [p358]

E. J. McCluskey. Minimization of boolean functions. The Bell System Technical Journal, 5:1417-1444, 1956. [p357]

W. V. O. Quine. The problem of simplifying truth functions. The American Mathematical Monthly, 59(8): 521-531, 1952. [p357]

W. V. O. Quine. A way to simplify truth functions. The American Mathematical Monthly, 62(9):627-631, 1955. [p357]

R Core Team. R: A Language and Environment for Statistical Computing. R Foundation for Statistical Computing, Vienna, Austria, 2016. URL http: //www. R-project.org/. ISBN 3-900051-07-0. [p]

C. Ragin. The Comparative Method. Moving beyond Qualitative and Quantitative Strategies. University Of California Press, Berkeley, Los Angeles \& London, 1987. [p357] 
C. Ragin. Fuzzy Set Social Science. University of Chicago Press, Chicago and London, 2000. [p361]

C. C. Ragin and J. Sonnett. Between complexity and parsimony: Limited diversity, counterfactual cases, and comparative analysis. In S. Kropp and M. Minkenberg, editors, Vergleichen in Der Politikwissenschaft. VS Verlag für Sozialwissenschaften, 2005. URL https://doi .org/10. 1007/9783-322-80441-9_9. [p359]

W. Stiehl. Truth Table Logic Optimizer, 2016. URL https://CRAN. R-project. org/package=Logicopt. R Package Version 1.0.0. [p366]

A. Thiem and A. Dușa. Introducing the QCA Package: A Market Analysis and Software Review. Qualitative E Multi-Method Research, 10(2):45-49, 2012. [p364]

\section{Adrian Dusa}

University of Bucharest, Department of Sociology

Soseaua Panduri nr. 90-92, sala 008, Bucharest sector 5

Romania

dusa. adrian@unibuc. ro 